\title{
Using an oilseed rape $x$ wild/weedy relative gene flow index for the monitoring of transgenic oilseed rape
}

\author{
Y. Devos ${ }^{1}$, A. De Schrijver ${ }^{2}$ and D. Reheul ${ }^{1}$ \\ ${ }^{1}$ Department of Plant Production, Faculty of Bioscience Engineering, Ghent University, Coupure Links 653, 9000 Ghent, Belgium \\ ${ }^{2}$ Division of Biosafety and Biotechnology, Scientific Institute of Public Health, Juliette Wytsmanstraat 14, 1050 Brussels, Belgium \\ Correspondence to: Yann Devos, Tel.: +32-9-264.60.99, Fax: +32-9-264.62.24, E-Mail: Yann.Devos@UGent.be
}

Received: August 1, 2007

Key words: Introgressive hybridisation, oilseed rape, post-market environmental monitoring, vertical gene flow, wild/weedy relatives.

\begin{abstract}
To estimate the introgressive hybridisation propensity (IHP) between transgenic oilseed rape and certain of its crosscompatible wild/weedy relatives at the landscape level, a conceptual approach was developed. A questionnaire was established enclosing the successive steps to successfully achieve introgressive hybridisation. Each step was described and scored, resulting in an IHP value for each cross-compatible wild/weedy relative. This approach revealed that in Flanders (Belgium) Brassica rapa has the highest IHP value, followed by Hirschfeldia incana, Diplotaxis tenuifolia, Raphanus raphanistrum and Sinapis arvensis. Using these values, monitoring priorities can be defined within the pool of cross-compatible wild/weedy oilseed rape relatives. It is discussed how the numerical quantification may serve as a valuable tool in case-specific monitoring and general surveillance of transgenic oilseed rape.
\end{abstract}

\section{Introduction}

The escape of transgenes into wild and weedy plant species is one of the concerns raised by the deliberate release into the environment of genetically modified (GM) crops, as the fitness of cross-compatible recipient plants may be altered by the acquisition of a transgene. Theoretically, and depending on which transgene is involved, altered fitness may enable wild/ weedy relatives to go extinct, to exacerbate a weed problem or to expand and invade new habitats, with unwanted effects on other species and agro-ecosystem integrity.

\section{Conceptual approach}

To estimate the IHP between GM oilseed rape and certain of its cross-compatible wild/weedy relatives at the landscape level, a questionnaire was established enclosing the successive steps to successfully achieve introgressive hybridisation: wild/ weedy relatives and oilseed rape (1) should co-occur, (2) have overlapping flowering periods, (3) be compatible and produce viable and fertile hybrids, (4) form viable and fertile backcross progeny, and (5) lead to the stable introgression of the transgene in the genome of the wild/weedy relative. Finally, the transgene (6) should persist in natural/weedy populations. Information on step 2 to 6 was retrieved from literature and reviewed for oilseed rape relatives found in Belgium (Devos et al., 2004 and 2007). Next to flowering characteristics, focus was put on hybridisation frequencies, fitness parameters of hybrids and their progeny, the transgene and on management practices, as these all play a crucial role in achieving introgression. Each step was described and scored, resulting in an IHP value for each cross-compatible wild/weedy relative in the studied region. Based on the IHP values, a relative ranking of wild/weedy relatives was established.

\section{Results}

Surveys in Belgium have shown that eleven cross-compatible species were found in areas where oilseed rape is grown, albeit their absolute abundances are unknown. From the eleven studied wild/weedy oilseed rape relatives, Brassica rapa showed the highest introgressive hybridisation propensity (rank 1), followed by Hirschfeldia incana (2). Due to their relative high abundance in Flanders, Diplotaxis tenuifolia, Raphanus raphanistrum and Sinapis arvensis were ranked in third position. Being less abundantly distributed Brassica nigra (6) was classified as fourth. Despite their potential to produce low numbers of inter-specific hybrids under field conditions, the IHP of 
Brassica juncea and Brassica oleracea (7) was low: both species are rare in Flanders and partial inbreeders. Low IHP values were also obtained for Sinapis alba (7), Diplotaxis muralis (10) and Erucastrum gallicum (11). Due to regional differences in distribution and abundance of wild/weedy relatives in the zones of co-occurrence, IHP values will vary regionally.

\section{Discussion}

Ranking wild/weedy relatives according to their IHP values offers the possibility to characterise monitoring priorities within the pool of cross-compatible wild/weedy relatives. On the one hand, knowledge gaps can be identified per wild/weedy relative. On the other hand, representative regions where potential adverse effects are most likely to be detected can be defined. By overlaying the geographical distribution maps of agricultural fields and wild plant species with geographic information system software, regions of co-occurrence can be identified and their frequency of occurrence be defined at the landscape level for successive years. As such, the location and number of regions to be followed up, and their distribution can be set to ensure adequate spatial and temporal replications in case-specific monitoring.

Since general surveillance aims at tracing unforeseen and cumulative changes related to the large-scale growing of transgenic crops, any unexpected shift in the abundance of wild/weedy relative plant populations in and outside agricultural habitats should be surveyed. At European level, the collaboration with existing surveillance networks is currently suggested, since these networks may provide good baseline data for comparison purposes. Farm-based surveys using farmers' questionnaires may deliver first hand data on the occurrence and relative abundance of wild/weedy relatives within agricultural fields. Networks that survey wild plant species may supply data on wild plant species in non-agricultural habitats. However, botanical survey networks seem to be of limited help for general surveillance in Belgium. Most programmes are not designed to characterise variation in populations or to distinguish 'unusual' from 'usual' variation at the landscape level. To suit the collected data to general surveillance needs, the abundance of wild oilseed rape relatives should be surveyed with a higher spatial accuracy and on a more frequent and systematic basis. Considering that monitoring should be costeffective and proportionate to the extent of market introduction of GM crops, survey activities could concentrate on regions where exposure is the greatest and conservational significance is the highest.

\section{References}

Devos, Y., De Schrijver, A. and Reheul, D. (2007) Quantifying the introgressive hybridisation propensity between transgenic oilseed rape and its wild/weedy relatives at the landscape level: implications for monitoring. Environmental Monitoring and Assessment (submitted).

Devos, Y., Reheul, D., De Schrijver, A., Cors, F. and Moens, W. (2004) Management of transgenic herbicide-tolerant oilseed rape in Europe: a case study on minimizing vertical gene flow. Environmental Biosafety Research 3:135-148.

To access this journal online: http://www.birkhauser.ch/JVL 